\title{
Design and construction of a DNA origami drug delivery system based on MPT64 antibody aptamer for tuberculosis treatment
}

\author{
Reza Ranjbar ${ }^{1}$, Mohammad Sadegh Hafezi-Moghadam²
}

${ }^{1}$ Ph.D., Molecular Biology Research Center, Baqiyatallah University of Medical Sciences, Tehran, Iran

${ }^{2}$ M.Sc., Department of Biotechnology, Faculty of Advanced Sciences and Technologies, University of Isfahan, Isfahan, Iran

\section{Type of article: Original}

\begin{abstract}
Introduction: With all of the developments on infectious diseases, tuberculosis (TB) remains a cause of death among people. One of the most promising assembly techniques in nano-technology is "scaffolded DNA origami" to design and construct a nano-scale drug delivery system. Because of the global health problems of tuberculosis, the development of potent new anti-tuberculosis drug delivery system without cross-resistance with known antimycobacterial agents is urgently needed. The aim of this study was to design a nano-scale drug delivery system for TB treatment using the DNA origami method

Methods: In this study, we presented an experimental research on a DNA drug delivery system for treating Tuberculosis. TEM images were visualized with an FEI Tecnai T12 BioTWIN at $120 \mathrm{kV}$. The model was designed by caDNAno software and computational prediction of the 3D solution shape and its flexibility was calculated with a CanDo server.

Results: Synthesizing the product was imaged using transmission electron microscopy after negative-staining by uranyl formate.

Conclusion: We constructed a multilayer 3D DNA nanostructure system by designing square lattice geometry with the scaffolded-DNA-origami method. With changes in the lock and key sequences, we recommend that this system be used for other infectious diseases to target the pathogenic bacteria.

Keywords: DNA, nanomedicine, nanostructures, drug delivery Systems, tuberculosis
\end{abstract}

\section{Introduction}

TB is a contagious disease that threatens human health globally. One-third of the people in the world are infected with Mycobacterium tuberculosis (MTB), the pathogenic agent of TB (1). According to the World Health Organization (WHO), about 8.5 to 9.2 million new TB cases occur every year, and that number currently is increasing. About 1.5 million deaths are caused by TB annually (2). One of the most important factors that makes TB the leading infectious cause of death is multi-drug resistant (MDR)-M. tuberculosis, which is difficult to control (3-6). Because of the global health problems of TB, the increasing rate of MDR- M. tuberculosis, and the high rate of co-infections with HIV, the development of potent new anti-TB drugs without cross-resistance with known antimycobacterial agents is urgently needed (7). MPT64 (or protein Rv1980c) protein is a highly specific protein secreted by the MTB complex, which includes M.tuberculosis, M. bovis, and M. africanum. This protein could be found with active Mycobacteria cells and the pathogenic Mycobacteria genus. MPT64 or protein Rv1980c is expressed only from M. tuberculosis, which means by existance of this relatively unique expression profile, this protein could be the diagnostic of choice for detecting active tuberculosis infections (8). To cure a patient who suffers from tuberculosis by anti-microbial treatment, the patient must take at least six months of chemotherapy. Because of the vague population of resistant $\mathrm{M}$. tuberculosis bacilli today, drugs have poor results in curing the patients (8). Therefore, drug delivery systems are important because they can increase the effectiveness of the chemotherapy. One of the best new methods to treat multi-drug strains of

\section{Corresponding author:}

Mohammad Sadegh Hafezi-Moghadam, Department of Biotechnology, Faculty of Advanced Sciences and Technologies, University of Isfahan, Isfahan, Iran. Tel: +98.9357480748, E-mail: sa.hafezi@gmail.com

Received: July 14, 2015, Accepted: November 24, 2015, Published: February 2016

iThenticate screening: November 24, 2015, English editing: January 14, 2016, Quality control: February 06, 2016 (C) 2016 The Authors. This is an open access article under the terms of the Creative Commons Attribution-NonCommercialNoDerivs License, which permits use and distribution in any medium, provided the original work is properly cited, the use is noncommercial and no modifications or adaptations are made. 
M. tuberuclosis is the use of drug carriers. A drug carrier should safely deliver the drug to the right place at the right time. Thus, it must have the ability to target the pathogenic bacteria and release the correct dosage of the drug where it is needed. DNA has been used for rational design and construction of nano-scale objects for about 30 years. The "DNA origami" method first was proposed and implemented by Paul W. K. Rothemund in 2006 (10). In this method, the designed nanostructure is created by folding a long viral single strand DNA (ssDNA) molecule. Several researchers have tried to develop a method to design a self-assembled DNA nanostructure. One of the most important was the attempt by Yan et al. that reported information about creating a nano-array DNA structure. The structure was made by a long scaffold that was shaped using some shorter strands (11). The problem was that they were unable to control the shape and size of the structures. Another advanced development was published by Shih et al., who reported a selffolding octahedron made by a long ssDNA and a group of smaller strands (12). They also suggested that self-folding RNA molecules could be used to create self-assembled 3D nanostructures, and they called the process "RNA origami" (13). The term origami comes from the Japanese art of creating special 3D shapes by folding paper, since the structure in this method is made by folding an ssDNA into the designed structure with smaller pices of ssDNA holding the structure together (14). The individual DNA origami structures typically range from approximately 50 to $400 \mathrm{~nm}$ in length, depending on the cross sectional geometry of the structure (15). DNA origami structures are created using a long, single-stranded "scaffold" and many short, single-stranded "staples." At any position in a DNA origami structure, one strand in the double-helix is contributed by the scaffold strand, which is a long circular piece of genomic DNA derived from the M13MP18 bacteriophage virus. The scaffold is around 7000-8000 bases in length and has a sequence that is fully known. The staple strands are much shorter, i.e., 30-50 bases in length, and they are designed to be piecewise complimentary to the sections of the scaffold strand that may be distant along the primary base sequence. During self-assembly, the scaffold must fold in order to spatially collocate sections of the scaffold that bind to a single staple. Hence, the staple sequences determine the scaffold's folding pattern. "Staple" strands are a group of small DNA strands that are complementary to pieces of the long ssDNA. These staples join the long pieces of the strands together to fold them into the desired 3D form. This manipulation "programs" the DNA to fold into a pre-designed geometry (16). Then, these folded strands are heat-annealed with a specific buffer with high concentration of magnesium to achieve the desired origami (17). DNA also can be one of the ideal materials to construct such devices. These structures can be designed to do tasks, such as sensing, computation, and actuation (17). Inspired by recent studies, we designed a three-dimensional DNA origami box that is capable of selectively interfacing with pathogenic bacteria and releases the drug near them. The purpose of this study was to design a nano-scale drug carrier that can find the threat and release one or more (up to three) drugs near the infected area to treat TB. Different threedimensional shapes of DNA structures were made to show that the complexity of these structures is endless (18-21). One of the most interesting articles about using this method for drug delivery systems described an autonomous DNA nano-robot that was capable of transporting molecular payloads to cells to treat cancer. They implemented several different logical AND gates and demonstrated their efficacy in selective regulation of the nano-robot's functions (22). In this article, for the first time, we tried to use the DNA origami method to design a nano-scale drug delivery system for the treatment of TB.

\section{Material and Methods}

Using a computer-aided design tool called caDNAno (23), we created a nano-robot. It is a square lattice structure that can be non-covalently fastened by staples modified with DNA aptamer-based locks. Designed oligonucleotides fold into its target shape during a thermal annealing ramp of rapid heating followed by slow cooling. Previously, DNA locks and DNA keys were used to control the opening of the lid on a DNA box (24). To operate our structure in response to pathogenic bacteria, we designed a DNA aptamer-based lock mechanism that opens the structure when the target ligand is detected. We designed two aptamer-complement duplexes on the left and right sides of the front of the nano-robot. For faster activation rates and keener sensitivity, shorter duplexes should be used, but this increased the frequency of unplanned activations. To attach the drugs into the nano-robot, they first must be modified. To achieve that goal, they should attach to a linker made of a 15-base ssDNA oligonucleotide by a covalent bond to the $5^{\prime}$ end of it. In the inner-face of the nano-robot and near the middle, there are three attachment sites designed to enable the places for different drugs and their linkers to attach to the nano-robot. The sites are staple strands with $3^{\prime}$ extensions that attach to the linker sequence by complementary effect. The model can be described as two caps made from DNA with drugs loaded between them, just two hands can be used to trap something. These caps lock together with two pieces of DNA that react to a key combination (Figure 1). The nano-robot stay inactive until it encounters the correct combination of ligand key sequences. When the keys are present, they open the lock combinations. When it is unlocked the nano-robot opens, allowing the loaded drugs to bind with the bacteria's surfaces and perform the desired function (Figure 2). The idea of our lock system came from Dr. Shawn Doglas' work, and he inspired it from aptamer beacons 
(25) and switching aptamers (26), which were studied for target-induced switching between an accompaniment duplex and a target complex, both of which were made of aptamers. As soon as both aptamers recognize their targets, the lock duplexes dissociate by attaching one of the strands to the key. Then, the caps are released, acting as an entropic spring, and they try to reconfigurate to turn into its previously sequestered surfaces (Figure 3 ). The payload inside the nano-robot was premodified by covalent attachment to the 5' end of a 15-base single-stranded DNA oligonucleotide linker. In the middle of the nano-robot, two inner attachment sites were arranged for the payload.

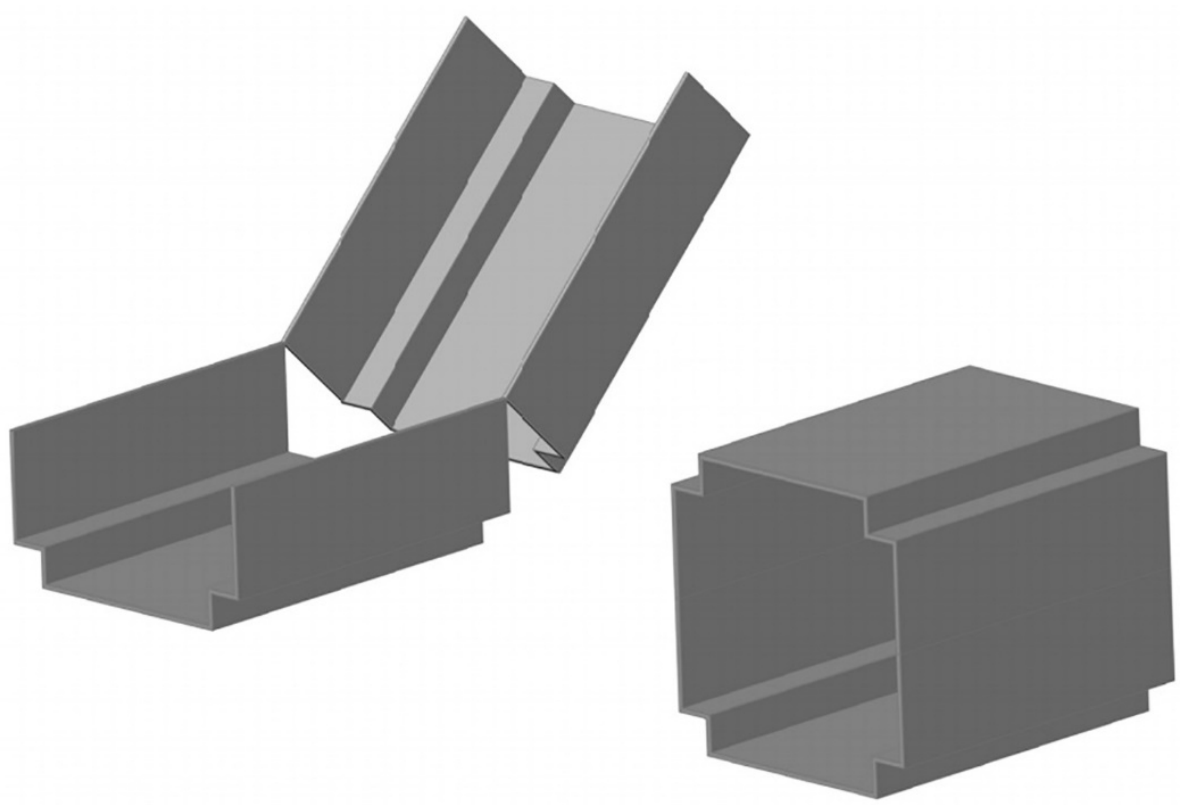

Figure 1. View of nano-robot in inactive (right) and active (left) mode, with the right key nano-robot activated and opened so the active site of the drug can interact with defected cells.

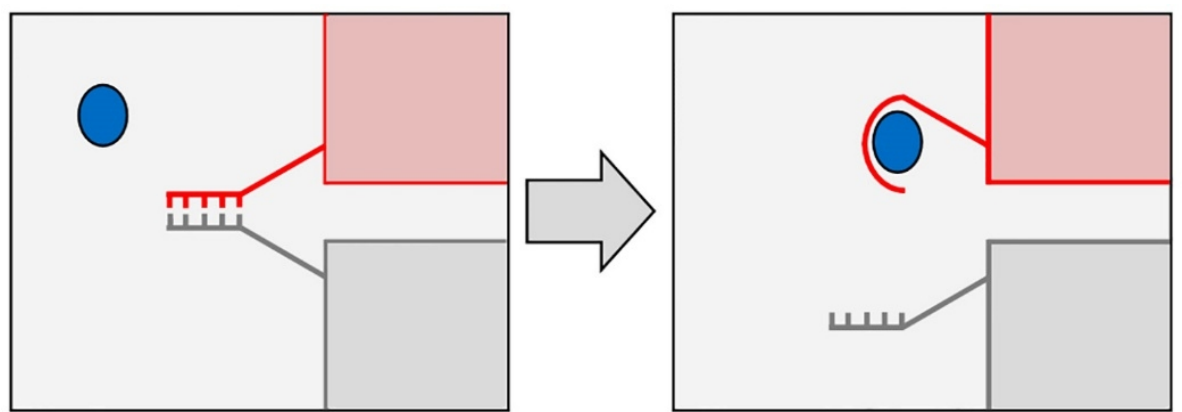

Figure 2. Aptamer lock mechanism, that include DNA aptamer strand (blue) and a partially complementary strand (gray). (A) These two DNA strands hold two caps together and keep the nano-robot deactivated. With right key that may be an antigen (red) the lock can be stabilized in a dissociated state and active the nano-robot.

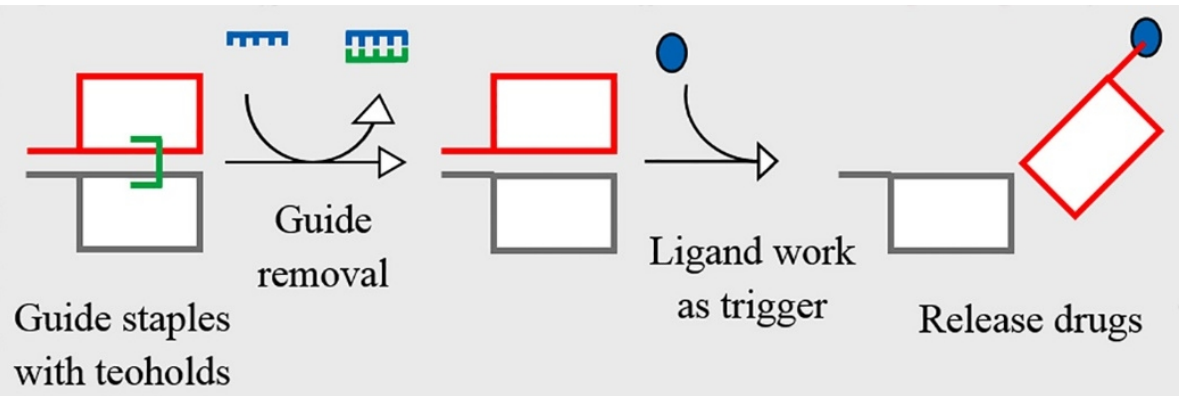

Figure 3. Side view shows guide staples (green) bearing 8- base toeholds help assembly of nano-robot. After folding, guide staples are removed by addition of fully complementary piece of DNA 
The sites are staple strands with 3' extensions that are designed to attach the linker by the complementary sequence. In constructing a "spring-load" device, it is important to ensure high-yield assembly. Two 8-base staples were used to attach the caps temporarily to keep the nano-robot from opening until the lock sequences bond to each other. These staples are called "guides," and they can be removed after the nano-robot is completely folded. Two keys were designed for the nano-robots to verify that they were not activated by a non-ligand-based mechanism. To activate the nano-robot, both locks must be opened together. The robot remains inactive when only one of the two locks is opened. The robot could be programmed to activate in response to a single type of key by using the same sequence of aptamers in both locks and, for that matter, by using two types of keys. Classical approaches to target that kind of signatures is the use of peptides, proteins, and mainly antibodies. Recent studies have shown that oligonucleotides, known as aptamers, can be used for this purpose with the same capacity. Complex, three-dimensional structures can be formed by DNA or RNA. These folded, short, single-stranded oligonucleotides could be designed to target specific ligands. Aptamers have a huge potential for developing biosensor devices and microbial drug delivery. First, a useful aptamer for detecting and targeting pathogens must be isolated. One of the best methods to isolate an aptamer for a specific target is to use random oligonucleotide sequence libraries in vitro-based screening process that is called Systematic Evolution of Ligands by Exponential enrichment (SELEX). For selecting aptamers with the best specificity and yield for a target protein, 11 aptamer candidate sequences were secured (Table 1). Still, no identical sequences were found in the results. After sequences for locks were determined and with staples sequences, the assembly process could be started. In this one pot process reaction, these single-stranded oligonucleotides combined rapidly with heating, and, after that, they cooled slowly. This cooling process should be done in four stages, and the cooling should be slower each time. With this heat treatment, all staples and scaffold strands attach to their complementary sequences, and the structure folds into the predicted shape. To achieve that goal, the mixture and temperatures are quite sensitive, and must be controlled carefully. The batch started with a buffer and salts. It should contain $2 \mathrm{mM} \mathrm{EDTA,} 6 \mathrm{mM}$ Tris (pH 8 at $20{ }^{\circ} \mathrm{C}$ ), $17 \mathrm{mM} \mathrm{MgCl}_{2}$ and heated to $85{ }^{\circ} \mathrm{C}$. For thermal annealing the mixture, it should be cooled to $60{ }^{\circ} \mathrm{C}$ in 85 min. For the second stage, it was cooled to $40{ }^{\circ} \mathrm{C}$ over a $30-\mathrm{hr}$ period. In the third stage, it was cooled to $30{ }^{\circ} \mathrm{C}$ in 50 hr. Finally, it was cooled to $24{ }^{\circ} \mathrm{C}$ in $100 \mathrm{hr}$. For the imaging process, the samples should be adsorbed onto glowdischarged grids for $5 \mathrm{~min}$ and treated with $0.5 \mathrm{M} \mathrm{MgCl}_{2}$. Then, the objects were stained with $2 \%$ uranyl formate and about 1 min with $25 \mathrm{mM} \mathrm{NaOH}$. Now, it was observed with an FEI Tecnai T12 BioTWIN at $120 \mathrm{kV}$.

Table 1. Aptamer sequences. Gibb's energy $(\Delta \mathrm{G})$ and melting temperature (Tm) of each sequence were calculated by Mfold for predicted secondary structures. Guanine contents of identified 24-nt regions were shown

\begin{tabular}{|l|l|l|l|l|}
\hline Name & Sequence & $\Delta \mathrm{G}(\mathrm{kcal} / \mathrm{mol})$ & $T_{\underline{m}}\left({ }^{\circ} \mathrm{C}\right)$ & $\mathrm{G}(\%)$ \\
\hline MPT64-A1 & CGGCACCCCGTCGCTATGTTGACC & -2.02 & 50.6 & 25 \\
\hline MPT64-A2 & CCCATATGCGCATCAAGATCTTCAT & -2.6 & 46.5 & 64 \\
\hline MPT64-A3 & GGGAGGGGCGGCGAAGGAGTGGCG & -2.5 & 50.7 & 67 \\
\hline MPT64-A4 & GGCGGAGGGGTTCGGGGTTGGCGC & -1.8 & 44.3 & 63 \\
\hline MPT64-A5 & GGGGGGGTGGCATTTTGGGGTGGG & 0.53 & 17.9 & 67 \\
\hline MPT64-A6 & GGGAGCAAATCCGGAATGTGGGGC & -3.01 & 57.6 & 46 \\
\hline MPT64-A7 & GGGGTTAGGCGAGGGGGGTGGGTG & 1.4 & -8.3 & 71 \\
\hline MPT64-A8 & GGGGATAGCAGGACAATGAGGGGG & -0.1 & 26.6 & 54 \\
\hline MPT64-A9 & GGGCGGGGGGGGATTCCGAGGCGC & -2.98 & 55.3 & 63 \\
\hline MPT64-A10 & GGGGATAGCAGGACAATGAGGGGG & -1.3 & 38.4 & 54 \\
\hline MPT64-A11 & CGGACGGTAGGGAAGGGGGGGGCG & 0.8 & 24.9 & 67 \\
\hline
\end{tabular}

\section{Results}

To design the nano-robot, a square lattice was chosen due to its size and stability, whereas, for larger structures, the honeycomb lattice was preferred, and, for the heavy strand, m13mp18 was chosen. After the strands' sequences were calculated, two strands were used as the linker to be attached in the middle of each cap. These strands were 15 bp $3^{\prime}$ DNA with the complementary sequence of the linker. The sequences chosen for the locks should add to the ends of each cap for specific targeting. To create the model with the CanDo server (21), first, the model should be created with caDNAno software. CaDNAno's results in designing the DNA are shown in Figure 4. After the sequences and structure were found, the CanDo server was used to create the model and the 3D shape of the nano-robot (Figure 5). It was confirmed that the designed shape remained stable at the conditions of the human body, and it should be a stable structure for carrying drugs and activating them near the target. After the simulation was completed, we constructed the carrier, and it was imaged by TEM (Figure 6). 

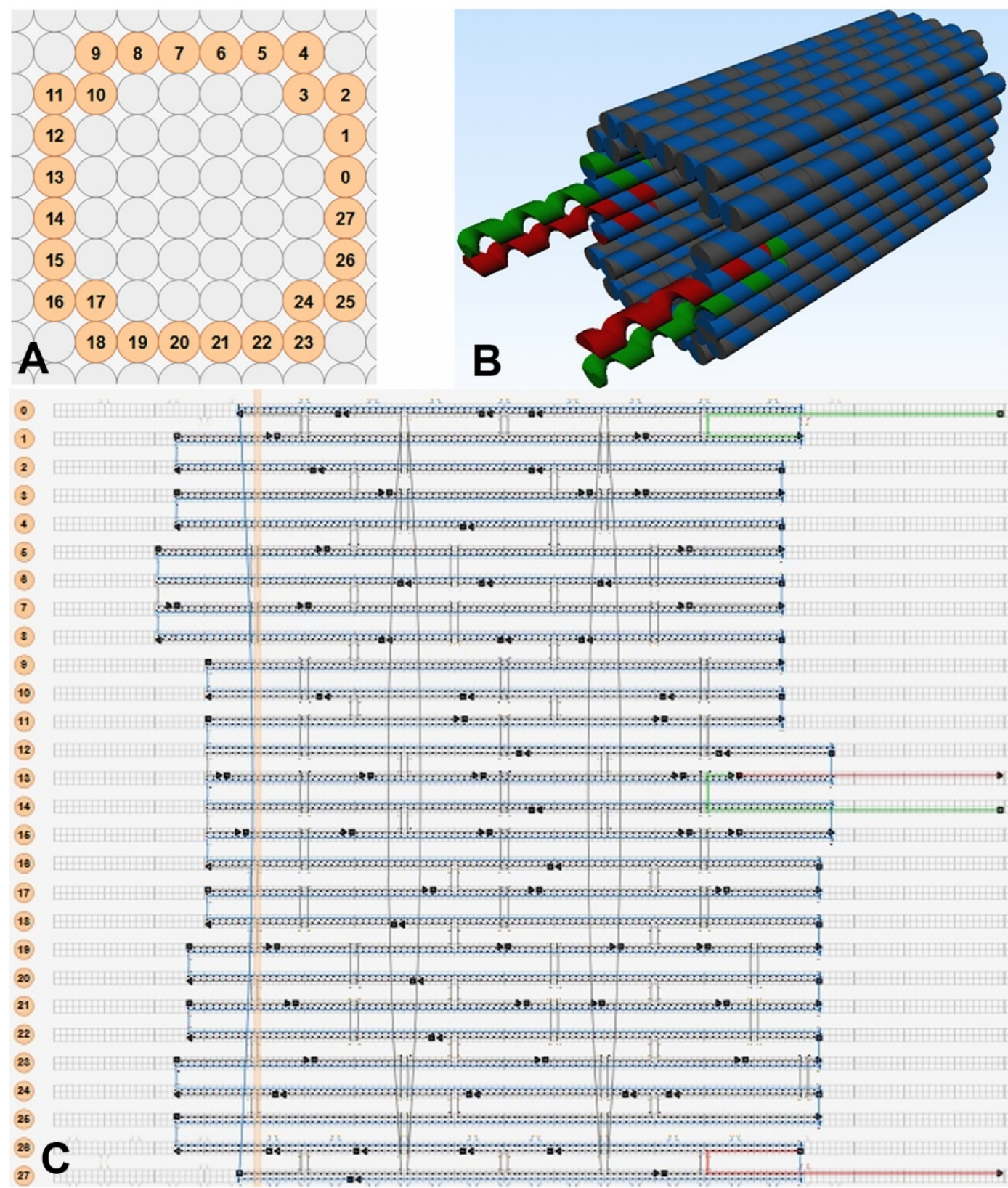

Figure 4. Results of caDNAno software: (A) Map of square lattice for the nano-robot; (B) 3D shape of nano-robot; (C) Map of DNA strands and sequences that can assemble the nano-robot
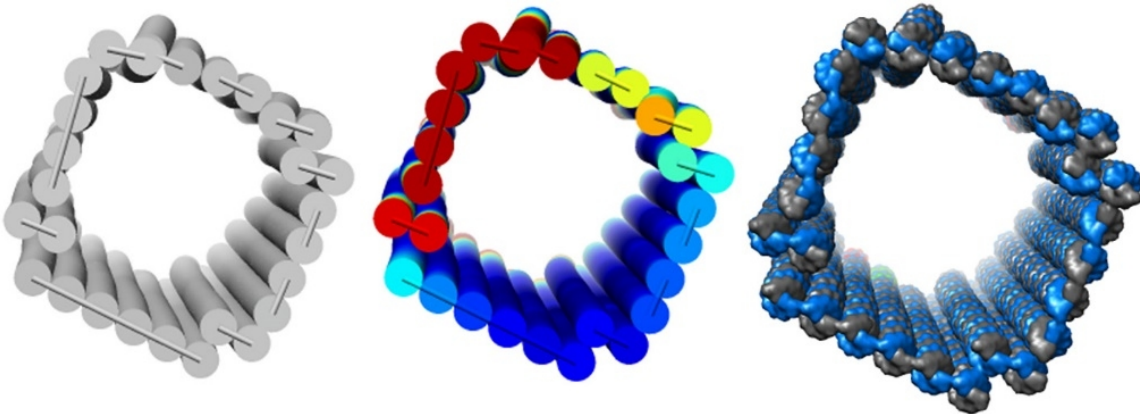

Figure 5. Results of CanDo server: (left) 3D shape of nano-robot; (middle) FEM model of structure; (right) 3D DNA model of nano-robot 


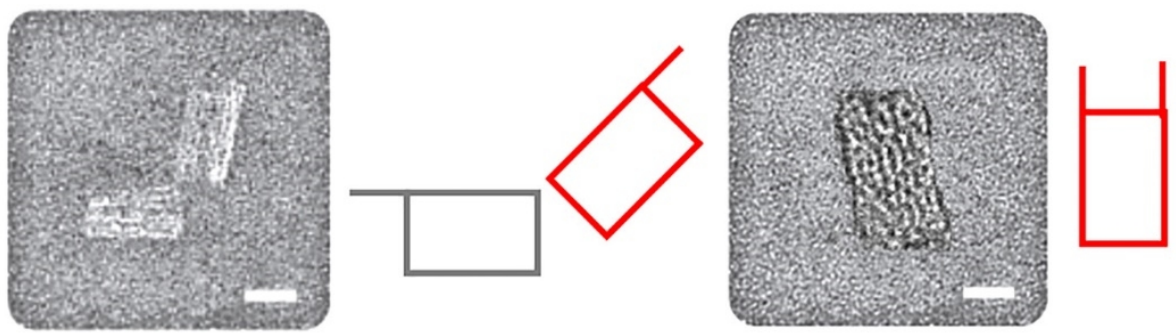

Figure 6. TEM images of nano-robot in closed and open conformations: Left: activated nano-robot from side; Right: inactive nano-robot from top

\section{Discussion}

In a related article, we found that shorter duplexes gave better sensitivity and faster activation rates, but at a cost of increased spontaneous activation (22). Among reported lengths for lock duplex, $24 \mathrm{bp}$ was found to be more reasonable (22), because it has shown similar sensitivity to a shorter duplex, such as $16 \mathrm{bp}$, that was activated at $10 \mathrm{pM}$. The length of the 24 bp lacks the unacceptable loss in sensitivity of longer duplexes, such as 30, 37, and 44 bp (activation at $1 \mathrm{nM}$ ). To create container-like structures using DNA origami, one may fold single layers of helices (10). For space filling shapes, the multi-layer approach often could be used (19). Single-layer objects assemble in a few hours, and the yield is nearly $100 \%$, but multi-layer objects take more time, as much as a week, and, according to their structure, the yield may be in the range of 5 to $20 \%$. Another difference between multi-layer and single-layer objects is their salt requirement. According to the base shape selected for the structure, one may close-pack DNA double-helical domains onto a square lattice or onto a honeycomb lattice. The term 'square lattice' means that each double-helical domain can have four neighbors, whereas only three neighbors are present in the honeycomb lattice (22). Every double-helical domain belongs to a particular lattice position by anti-parallel strand crossovers along the helical axis that connects to the next double-helical domains. The spacing of crossovers depends on the lattice packing. Every B-form DNA double helical twists $360^{\circ}$ per $10.4 \mathrm{bp}$. In order to close-pack DNA double-helical domain onto a square lattice, cross-overs must be placed to the four nearest neighbors, which are arranged in four-fold symmetry. Constant cross-over spacings of $21 \mathrm{bp}$ among particular pairs of neighboring helical domains exist when the native B-form DNA geometry is applied. Therefore, an average spacing of $21 / 4=5.25 \mathrm{bp}$ will be reached when cross-overs to the other three neighbors in a square lattice are examined. Irregular cross-over spacing intervals must be used to achieve this. A square lattice packing with constant cross-over spacing intervals could be achieved by assuming an average helicity of 10.67 bp per turn in B-form DNA. The backbone of a strand rotated by $270^{\circ}$ in intervals of $8 \mathrm{bp}$ results in a fourfold symmetry. Applying cross-overs to four neighbors in 32-bp intervals in a four-fold symmetry directs us to intervals of 8 bp in a square lattice packing, causing underwinding of every double-helical domains from the natural $10.5 \mathrm{bp}$ to the applied 10.67 bp per turn. This will result in twisting torques transmitted by cross-overs. The entire object will be twistdeformed globally by the imposed internal torques. In order to eliminate the global twist for objects in square-lattice packing, constant 8 bp spacing between cross-overs should be departed towards achieving effective double-helical twist densities closer to natural $10.5 \mathrm{bp}$ per turn. It was found that in $10.4 \mathrm{bp}$ per turn case, elimination of global twist occurs $(18,22)$. Creation of large torsional stiffness in the helical direction can also minimize the global twist in multilayer square lattice objects. In the case of single-layer square lattice DNA origami objects, the constant 16 bp spacing between cross-overs to neighboring double-helical domains causes a twisted shape in solution. The twist deformation might be abolished if adhesion interaction with surfaces occurs. The result would be objects lying flat on a surface (20). Electrostatic immobilization can eliminate the twist deformation for single-layer DNA origami objects through surface deposition. Therefore, densely-packed objects with rectangular features might be created by eliminating the existing global twist deformation.

Straight and, at the same time, more porous structures are created by the honeycomb lattice packing. Connections of double-helical domains in a DNA origami object could be restored better by both staple and scaffold strands. Two cross-over reference frames that are shifted in the helical direction by 5 bp or 6 bp could be defined by accommodating both staple and scaffold cross-overs. No global deformation would occur for multi-layer objects with cross-sectional aspect ratios around 1 or sufficient thickness, keeping in mind that major and minor groove in B-form DNA have been neglected in this approach. In order to avoid undesired rolling up of thinner objects, major and minor groove phosphate position should be aware of. Also, this could be achieved by working with high densities of staple cross-overs rather than scaffold cross-overs as much as possible. The dimensions of a DNA origami object might be estimated by rule 
of thumb as follows: The length of double-helical domains is calculated via $\mathrm{N}^{*} 0.34 \mathrm{~nm}$, where $\mathrm{N}$ is the number of base pairs in the double-helical domain. Both for a single-layer square lattice and a multi-layer honeycomb lattice, objects with the value of $0.34 \mathrm{~nm}$ per bp hold true. To calculate the width of a square lattice nano-robot along the horizontal or vertical cross-sectional axis, this formula could be used: " $2 \mathrm{H}+(\mathrm{H}-1) \mathrm{g}$," where $\mathrm{H}$ is the number of double helical domains that have a width of $2 \mathrm{~nm}$ and there is a gap between cross-overs that is called the inner helical gap, which is $g$ in the formula $(16,27,28)$. To create a desired cuboid shape, first the size of the cargo must be known. After that, the length of each DNA helix can be calculated. Now, the number of helices per layer must be decided. With this information, the dimensions of the nano-robot are known, and it can be designed. One of the limitations in designing for the maximum potential size of nano-robot is the length of the scaffold strand. To minimize undesired multimerization, we used unpaired scaffold bases at the ends of the helices. If the scaffold strand has a circular loop topology, a long, unpaired loop should be used to span the distance between the starting and ending points (20). Staple strands are attached to the neighboring helices in cubic manner at the locations of two helices twist near each other in the intersection planes. These cross-overs can be seen on the 2D scheme (Figure 4c) as thin lines and that is where the phosphate backbones connect to each other (21). Finally, after placing every staple, the Watson crick complementary sequences can be determined for the staples. With these sequences, the construction of the nano-robot was started. After the construction process was completed, TEM should approve that the nano-robot was constructed as planned (23).

\section{Conclusions}

We succeeded in designing and constructing square lattice 3D DNA nano-robot nanostructures using the scaffoldedDNA-origami method. Since the density of the material in the square lattice is higher than honeycomb, we expect that the stability of the square lattice objects should be greater and more resistant to compression. Drug carriers are used to decrease the side effects of drugs used to destroy pathogenic bacteria thereby making the bacteria more targetable. DNA is one of the best materials for drug delivery nanostructures. There are several techniques to design and synthesize DNA nanostructures. One of the most promising assembly techniques in nano-technology is 'Scaffolded DNA origami.' These objects can be more than simple nanostructures and have different functions to increase targeting. In this article, we explained the details one needs to know to design a targetable DNA origami nano-robot. The descriptive report of our design is enclosed.

\section{Acknowledgments:}

This work was supported by the Baqiyatallah University of Medical Sciences. We thank our colleagues at the Molecular Biology Research Center, Baqiyatallah University of Medical Sciences and University of Isfahan, who provided insight and expertise that greatly assisted the research.

\section{Conflict of Interest:}

There is no conflict of interest to be declared.

\section{Authors' contributions:}

Both authors contributed to this project and article equally. Both authors read and approved the final manuscript.

\section{References}

1) Chen F1, Zhou J, Luo F, Mohammed AB, Zhang XL. Aptamer from whole-bacterium SELEX as new therapeutic reagent against virulent Mycobacterium tuberculosis. Biochemical and biophysical research communications, 2007. 357(3): 743-8. Doi: 10.1016/j.bbrc.2007.04.007, PMid: 17442275

2) Raviglione, M.C. The TB epidemic from 1992 to 2002. Tuberculosis, 2003. 83(1): 4-14. Doi: 10.1016/S14729792(02)00071-9

3) Mckinney, J.D., W.R. Jacobs, and B.R. Bloom, Persisting problems in tuberculosis. 1998, Academic Press.

4) Velayati AA, Farnia P, Merza MA, Zhavnerko GK, Tabarsi P, Titov LP, et al. New insight into extremely drug-resistant tuberculosis: using atomic force microscopy. European Respiratory Journal, 2010. 36(6): 1490-3. Doi: 10.1183/09031936.00064510, PMid: 21119210

5) Farnia P, Mohammad RM, Merza MA, Tabarsi P, Zhavnerko GK, Ibrahim TA, et al. Growth and cell-division in extensive (XDR) and extremely drug resistant (XXDR) tuberculosis strains: transmission and atomic force observation. Int J Clin Exp Med, 2010. 3(4): 308-14. PMID: 21072265, PMCID: PMC2971545

6) Khorvash F, Javadi AA, Izadi M, Jonaidi Jafari N, Ranjbar R. Spinal tuberculosis: a major public health hazard in Isfahan. Pak J Biol Sci., 2007. 10(19): 3400-4. PMid:19090158 
7) Tomioka, H., Current status of some antituberculosis drugs and the development of new antituberculous agents with special reference to their in vitro and in vivo antimicrobial activities. Current pharmaceutical design, 2006. 12(31): 4047-70. Doi: 10.2174/138161206778743646, PMid: 17100611

8) Zhu C, Liu J, Ling Y, Yang H, Liu Z, Zheng R, et al. Evaluation of the clinical value of ELISA based on MPT64 antibody aptamer for serological diagnosis of pulmonary tuberculosis. BMC Infect Dis. 2012. 12(1): 96. Doi: 10.1186/1471-2334-12-96, PMid: 22520654, PMCid: PMC3410803

9) Hampson SJ, Parker MC, Saverymuttu SH, Joseph AE, Mcfadden JJP, Hermon-Taylor J. Quadruple antimycobacterial chemotherapy in Crohn's disease: results at 9 months of a pilot study in 20 patients. Alimentary pharmacology \& therapeutics, 1989. 3(4): 343-52. Doi: 10.1111/j.1365-2036.1989.tb00221.x

10) Rothemund, P.W., Folding DNA to create nanoscale shapes and patterns. Nature, 2006. 440(7082): p. 297302. Doi: 10.1038/nature04586, PMid: 16541064

11) Yan H, LaBean TH, Feng L, Reif JH. Directed nucleation assembly of DNA tile complexes for barcodepatterned lattices. Proceedings of the National Academy of Sciences, 2003. 100(14): 8103-8. Doi: 10.1073/pnas.1032954100, PMid: 12821776, PMCid: PMC166189

12) Shih, W.M., J.D. Quispe, and G.F. Joyce, A 1.7-k ilobase single-stranded DNA that folds into a nanoscale octahedron. Nature, 2004. 427: 618-21. Doi: 10.1038/nature02307, PMid: 14961116

13) Williamson, J.R., RNA origami. Nature Structural \& Molecular Biology, 1994. 1(5): 270-2. Doi: 10.1038/nsb0594-270

14) Zadegan, R.M. and M.L. Norton, Structural DNA Nanotechnology: From Design to Applications. Int J Mol Sci. 2012. 13: 14. Doi: 10.3390/ijms13067149, PMid: 22837684, PMCid: PMC3397516

15) Rothemund, P., Scaffolded DNA origami: from generalized multicrossovers to polygonal networks. Nanotechnology: science and computation. Natural Computing Series. New York: Springer, 2006: p. 3-21.

16) LeHotan, C., Growth Quantification and Length Distributions of DNA Origami Polymers. 2013.

17) Nangreave, J., et al., DNA origami: a history and current perspective. Curr Opin Chem Biol. 2010. 14(5): 608-15. doi: 10.1016/j.cbpa.2010.06.182, PMID: 20643573

18) Douglas SM, Dietz H, Liedl T, Högberg B, Graf F, Shih WM. Self-assembly of DNA into nanoscale threedimensional shapes. Nature, 2009. 459(7245): 414-8. Doi: 10.1038/nature08016, PMid: 19458720, PMCid: PMC2688462

19) Dietz, H., S.M. Douglas, and W.M. Shih, Folding DNA into twisted and curved nanoscale shapes. Science, 2009. 325(5941): 725-30. Doi: 10.1126/science.1174251, PMid: 19661424, PMCid: PMC2737683

20) Ke Y, Douglas SM, Liu M, Sharma J, Cheng A, Leung A, et al. Multilayer DNA Origami Packed on a Square Lattice. J Am Chem Soc. 2009; 131(43):15903-8. doi: 10.1021/ja906381y. PMID: 19807088, PMCID: PMC2821935

21) Castro CE, Kilchherr F, Kim D, Shiao EL, Wauer T, Wortmann P, et al. A primer to scaffolded DN A origami. NATURE METHODS, 2011. 8: 9. doi:10.1038/nmeth.1570

22) Douglas, S.M., I. Bachelet, and G.M. Church, A logic-gated nanorobot for targeted transport of molecular payloads. Science, 2012. 335(6070): 831-4. Doi: 10.1126/science.1214081, PMid: 22344439

23) Douglas SM, Marblestone AH, Teerapittayanon S, Vazquez A, Church GM, Shih WM. Rapid prototyping of 3D DNA-origami shapes with caDNAno. Nucleic Acids Res. 2009. 37(15):5001-6. doi: 10.1093/nar/gkp436, PMID: 19531737, PMCID: PMC2731887

24) Andersen ES, Dong M, Nielsen MM, Jahn K, Subramani R, Mamdouh W, Self-assembly of a nanoscale DNA box with a controllable lid. Nature, 2009. 459(7243): 73-6. Doi: 10.1038/nature07971, PMid: 19424153

25) Hamaguchi, N., A. Ellington, and M. Stanton, Aptamer beacons for the direct detection of proteins. Analytical biochemistry, 2001. 294(2): 126-31. Doi: 10.1006/abio.2001.5169, PMid: 11444807

26) Nutiu, R. and Y. Li, In Vitro Selection of Structure - Switching Signaling Aptamers. Angewandte Chemie, 2005. 117(7): 1085-9. Doi: 10.1002/ange.200461848

27) Steinhauer C, Jungmann R, Sobey TL, Simmel FC, Tinnefeld P. DNA Origami as a Nanoscopic Ruler for Super - Resolution Microscopy. Angewandte Chemie International Edition, 2009. 48(47): 8870-3. Doi: 10.1002/anie.200903308, PMid: 19830751

28) Sanderson, K., Bioengineering: What to make with DNA origami. Nature, 2010. 464(7286): 158. Doi: 10.1038/464158a, PMid: 20220817 